\title{
La calidad en la docencia universitaria. Una aproximación desde la percepción de los estudiantes
}

\author{
JUAN MONTENEGRO ORDONEEZ* \\ Universidad Señor de Sipán - Perú \\ Recibido el 24-08-18; primera evaluación el 10-07-19; \\ segunda evaluación el 02-09-19; tercera evaluación; \\ 04-02-20; aceptado el 11-02-20
}

\section{RESUMEN}

El objetivo fue construir una noción de calidad de la docencia universitaria, en función a la demanda de los destinatarios del servicio: los estudiantes, a partir de sus opiniones y percepciones, contrastándola con las nociones que se desprenden o se consideran en la normativa respectiva, dada en el país. Se desarrolló en la Universidad Señor de Sipán, Universidad Privada Juan Mejía Baca y Universidad Nacional Pedro Ruiz Gallo. El diseño del estudio es exploratorio y descriptivo, así como de tipo fenomenológico hermenéutico y de corte transversal. La muestra estuvo constituida por 164 estudiantes. El 90\% concuerda en que una universidad de calidad, necesariamente, debe contar con docentes de calidad, e indica que este docente de calidad debe evidenciar dominio en cuatro dimensiones: dimensión temática, dimensión metodológica, dimensión científica, y dimensión ética, concordando con la literatura consultada.

Palabras clave: Calidad de la educación, docencia, universidades, enseñanza.

\footnotetext{
* Licenciado en Sociología por Universidad Nacional Pedro Ruiz Gallo (UNPRG). Magíster en Ciencias de la Educación con mención en Investigación y Docencia por la UNPRG. Docente invitado en la Facultad de Ciencias Histórico Sociales y Educación de la UNPRG, en el periodo 2006-2016, en el Programa de Licenciatura en Educación Modalidad Mixta (LEMM). Docente a tiempo completo en la Universidad Señor de Sipán (USS). Correo electrónico: juanmontenegro2000@gmail.com y jmonteneg@crece.uss.edu.pe
} 


\section{Quality in university teaching. An approximation from student's perception}

\section{Abstract}

The objective was to build a notion of quality of university teaching, depending on the demand of the recipients of the service, the students, based on their opinions and perceptions, contrasting it with the notions that emerge or are considered in the respective regulations, given in the country. It was developed at the Universidad Señor de Sipán, Juan Mejía Baca Private University and Pedro Ruiz Gallo National University. The study design has been exploratory and descriptive, as well as hermeneutical phenomenological type and cross-sectional. The sample consisted of 164 students. $90 \%$ agree that a quality university must necessarily have quality teachers, and indicates that this quality teacher must demonstrate mastery in four dimensions: thematic dimension, methodological dimension, scientific dimension, and ethical dimensión, according to the literature consulted.

Keywords: Quality of education, teaching, universities, teaching.

A qualidade no ensino universitário. Uma abordagem desde a percepçáo dos alunos

\section{Resumo}

O objetivo foi construir uma noção de qualidade do ensino universitário, dependendo da demanda dos destinatários do serviço, os alunos, com base em suas opiniôes e percepçóes, contrastando-o com as noçóes que emergem ou são consideradas nos respectivos regulamentos, Dado no país. Foi desenvolvido na Universidade Senhor de Sipán, na Universidade Particular Juan Mejía Baca e na Universidade Nacional Pedro Ruiz Gallo. O desenho do estudo foi exploratório e descritivo, bem como do tipo fenomenológico hermenêutico e transversal. A amostra foi composta por 164 estudantes. 90\% concordam que uma universidade de qualidade deve necessariamente ter professores de qualidade e indica que esse professor de qualidade deve demonstrar domínio em quatro dimensôes: dimensão temática, dimensão metodológica, dimensão científica e dimensão ética; de acordo com a literatura consultada.

Palavras chave: Qualidade da educação, ensino, universidades, ensino. 


\section{INTRODUCCIÓN}

La calidad es una variable compleja para definirla, básicamente, cuando se trata de servicios. Y la educación es un servicio que, actualmente, está siendo objeto de debate a fin de sumarse a la competencia por la calidad en la que participan los otros productos que oferta el mercado, en un mundo globalizado.

En este contexto, el presente artículo toma en cuenta la preocupación por ubicar a la educación universitaria como un servicio que también puede ser de calidad para satisfacer a sus usuarios: los estudiantes, por ello, tienen el objetivo de construir una noción de calidad de la docencia universitaria, en función a la demanda de los estudiantes, a partir de sus opiniones y percepciones, contrastándola con las nociones que se desprenden o se consideran en la normativa respectiva, dada en el país, así como, con la literatura consultada.

Una escuela es de excelencia cuando es capaz de ofrecer servicio de calidad en todos sus productos, básicamente, a los estudiantes y a la sociedad de donde ellos y ellas provienen. Un ciudadano está preparado si ha egresado de una escuela de calidad, por lo tanto, es capaz de enfrentarse y solucionar los problemas que la cotidianidad le presenta en sus diversas actividades laborales realizadas (Álvarez de Zayas, 2005).

Asimismo, la escuela debe proporcionar una formación integral con la finalidad de formar personas competentes para satisfacer las variadas demandas de una sociedad cada día más exigente. El autor lo denomina «encargo social». La didáctica universitaria, también denominado proceso enseñanza aprendizaje (PEA), o en otros contextos, proceso docente educativo (PDE), debe garantizar la inserción laboral de los egresados, a fin de contribuir a la mejora de la sociedad en su conjunto (Álvarez de Zayas, 2005).

En los últimos ańos, el Estado peruano ha tomado la iniciativa en mejorar la calidad del servicio educativo, tanto en el nivel básico como en el nivel superior, y, dentro de este, el nivel superior universitario. No obstante, no podemos hablar de calidad docente, si no consideramos la calidad educativa; es decir, la calidad docente está incluida en la calidad educativa, y quizá, sea el elemento más relevante.

\section{Marco teórico}

Iniciamos por aproximarnos a la definición de calidad, en términos generales, y luego, a la definición de calidad educativa, antes de llegar a la definición de la calidad docente encontrada en la literatura consultada. 


\subsection{La calidad}

La calidad está en función de alcanzar expectativas preestablecidas; esto es el grado de coherencia entre intención, acción y logro. Como veremos más adelante, la concepción de la calidad en el servicio educativo superior universitario, en Perú, encaja u obedece a este enfoque cuando se refiere a un "grado de ajuste» entre acción y propósito institucional (decreto supremo 016-2015MINEDU, 2015).

Otras nociones nos presentan a la calidad como una categoría que involucra diversos aspectos. Entre ellas está el político, económico, cultural, moral, y hasta el empresarial. Se entrecruzan intereses y expectativas de los vendedores de un producto y los consumidores del mismo. La calidad es una "categoría que está expuesta a subjetividades» (Sistema Nacional de Evaluación, Acreditación y Certificación de la Calidad Educativa [Sineace], 2016, p. 8); es «un concepto abstracto tan amplio en definición y aplicación que cada organización puede entenderlo desde sus propios intereses» (Traba et al., citados por Tumino y Poitevin, 2013, p. 64).

No tiene sentido evaluar o diagnosticar un servicio y un bien o manufactura, de la misma manera, porque el usuario o el cliente, en el caso de un servicio, es parte del proceso y la evaluación que realiza es subjetiva. Está en juego sus intereses, estado de ánimo, preferencias, experiencias, entre otros; por ello, existe la necesidad de conocer la opinión de los clientes o consumidores del servicio para evaluar su calidad; además que, en un servicio, se dan en el mismo momento, la producción, la adquisición y el consumo (Tumino y Poitevin, 2013). Es decir, en un servicio, solo los usuarios del mismo están en la capacidad y libertad de indicar o calificar si el servicio que reciben es o no de calidad. Ellos y ellas son los receptores del servicio, los que van experimentar los beneficios que le proporcionan, las satisfacciones que les genera, etc.

Calidad, entonces, no es sinónimo de perfección. No es un calificativo que, inequívocamente, se puede otorgar a un producto determinado sobre la base de estándares prestablecidos, sin considerar la percepción o punto de vista de los consumidores o usuarios (en el caso de los servicios). Va a depender de ellos y ellas, básicamente; será necesario de su intervención en las evaluaciones que se hagan en los procesos evaluativos de medición de la calidad, para determinar si corresponde o no, considerar de calidad, a un determinado producto o servicio (Sineace, 2016). 


\subsection{Calidad educativa}

Trasladar la noción de calidad al campo educativo genera otras situaciones que hacen más difícil la comprensión conceptual, porque, por ejemplo, puede verse desde dos ámbitos: la calidad como proceso de mejora, que alude al comportamiento institucional (autonomía, transparencia y efectividad); y la calidad como transformación individual, que refiere al comportamiento de estudiantes y docentes como copartícipes del proceso educativo (Castilla, 2011).

En Perú, se generó un espacio de reflexión y debate con la participación de expertos con el objetivo de construir una definición de calidad educativa. Después de un año, se arriba al siguiente postulado: «Hablar de calidad es aludir a la finalidad que se persigue, que en el caso de la educación es la formación de la persona» (Sineace, 2016, p. 6). Entonces, la mirada se dirige a los estudiantes, pues, en ellos y ellas, se verán los logros alcanzados o los resultados obtenidos, previamente definidos, a fin de aproximarse al nivel de la calidad de la educación en cuestión.

Más que concluir en una definición, se logró delimitar «aspectos importantes que deben considerarse en una aproximación a calidad educativa»; por ejemplo, el contexto, la finalidad e intereses involucrados (Sineace, 2016, p. 6).

Si el objetivo del docente es la formación de la persona, a fin de enfrentarse a los desafíos de la sociedad, y salir airoso cada día, entonces, esta persona formada es quien deberá dar cuenta si el proceso educativo del cual formó parte fue o no de calidad (Álvarez de Zayas, 2005).

Las múltiples definiciones existentes indican varios elementos, como la búsqueda constante del mejoramiento, la competencia técnica y la excelencia en la acción, pero todos ellos, relacionados con el cumplimiento de los propósitos educativos (Alvarado et al., 2016), concordando con la noción de calidad en la legislación peruana.

La legislación peruana contempla dos fines educativos: (1) Formar personas capaces de lograr su realización ética, intelectual, artística, cultural, afectiva, física, espiritual y religiosa, y (2) contribuir a formar una sociedad democrática, solidaria, justa, inclusiva, próspera, tolerante y forjadora de una cultura de paz que afirme la identidad nacional sustentada en la diversidad cultural, étnica y lingüística (Ley 28044, 2003).

El propósito de la educación peruana es formar personas integras y que estas contribuyan a la forja de una sociedad democrática, sustentada en la diversidad cultural. En consecuencia, en la medida en que los egresados de las instituciones educativas evidencien ser ciudadanos íntegros y contribuyentes a una sociedad democrática sostenida en la diversidad cultural, podemos hablar 
de una educación peruana de calidad. En esta oportunidad, se toma como referente la noción planteada en la normatividad vigente para el discernimiento de la calidad del servicio educativo.

Desde la Declaración de Bolonia, «se puso de manifiesto la relevancia de la evaluación y garantía de la calidad en los estudios universitarios, ya sean de grado o de posgrado" (Buela-Casal y Castro, 2011, p. 254); y en la Declaración de Berlín, «se recoge, como un aspecto esencial, el establecimiento de mecanismos de garantía de la calidad en la educación superior europea» (p. 254).

En este marco, emergen distintos modos u opciones no solo de concebir la calidad educativa, sino de medirla. Como bien lo plantean y citan Santelices, Galleguillos, González y Taut (2015, p. 2):

La discusión sobre calidad docente en la literatura internacional se había desarrollado, hasta recientemente, desde tres líneas principales de investigación: (a) el estudio de características observables del profesor que pueden tener un impacto sobre el desempeño de los alumnos (Hanushek \& Rivkin, 2006); (b) el análisis de calidad docente, definida como prácticas profesionales basadas en estándares profesionales y medidas usualmente por medio de pautas de observación de clases (Danielson, 2007; Darling-Hammond, 2012; Papay, 2012); y (c) estimaciones de valor agregado, basadas en el rendimiento de los estudiantes en pruebas estandarizadas (Millman, 1997).

En otros términos, la calidad educativa universitaria puede medirse desde las competencias profesionales del docente que afectan el desempeño de los estudiantes, desde su didáctica o práctica pedagógica en base a estándares de medición de esta, o desde el rendimiento de los estudiantes medidos con instrumentos estándares.

El presente estudio asume la calidad educativa como resultado de la percepción de los consumidores o usuarios del servicio; es decir, de los estudiantes, la misma que pasa por la calidad docente. Es insoslayable la presencia del docente en la medición de la calidad educativa. Es incoherente pretender certificar la calidad a un determinado servicio educativo si no está presente la calidad del docente. El docente es agente inherente a la medición de la calidad de todo servicio educativo.

\subsection{Calidad docente}

Quien ejerce la docencia universitaria es un profesional, pero no necesariamente un profesional de la pedagogía y la didáctica. No es un docente de carrera. No obstante, cuando es evaluado, se toma en cuenta estas dos grandes dimensiones: dominio temático, que tiene que ver con su formación profesional; 
y el dominio de la enseñanza, es decir, dominio metodológico. Debe ser un especialista en pedagogía y didáctica, a fin de poder lograr nuevos aprendizajes en sus estudiantes (Montero et al., 2017).

Un docente universitario debe tener un compromiso fundamental con el conocimiento (dominio temático), saber interactuar con el estudiante (buen trato), y formación pedagógica para lograr aprendizajes significativos en ellos (dominio metodológico) (Rizo, 1999).

Aparece una tercera dimensión, la cual es concomitante a las funciones de la universidad, en el mundo. Un docente universitario también debe ser un especialista en investigación científica. Se le exige que produzca nuevo conocimiento orientado a la solución de problemas contextuales específicos. Ello concuerda con la Conferencia Mundial sobre la Educación Superior (Unesco, 2009), cuando señala que la educación superior deberá liderar la creación de conocimientos en el mundo para enfrentar grandes retos mundiales, como la seguridad alimentaria, el cambio climático, la gestión del agua, el diálogo intercultural, las energías renovables y la salud pública. Igualmente, concuerda con la Declaración sobre la Ciencia y el uso del Saber Científico (Unesco, 1999), cuando declara que la investigación científica y la utilización del saber derivado de esa investigación deberá tener un solo propósito: lograr el bienestar de la humanidad.

Entonces, además de ser un especialista en su formación profesional, un docente universitario debe ser un especialista en pedagogía y didáctica, y, a su vez, un especialista en Investigación científica, la cual deberá proporcionar nueva información a fin de solucionar la problemática de la sociedad, quien demanda oportunas y efectivas atenciones.

Hasta podría hablarse de una cuarta dimensión que es transversal a las tres anteriores: la dimensión ética (ser un buen ciudadano). La formación actitudinal es parte del ejercicio docente, en tanto implica, práctica y enseñanza de valores: respeto, puntualidad, responsabilidad, honestidad, entre otros. Como anota Álvarez de Zayas (2005), «lo fundamental en la educación es formar al hombre: educarlo, desarrollarlo e instruirlo» (p. 46). En suma, la dimensión ética es concomitante al ejercicio de la docencia.

En este marco, la medición de la calidad del docente universitario sugiere tomar en cuenta estas cuatro dimensiones: dimensión temática, dimensión metodológica, dimensión científica, y dimensión ética. Las mismas contempladas en la legislación peruana, y también en la percepción de los estudiantes, así como en la literatura consultada.

Es necesario que la universidad reoriente su función social, básicamente, hacia la solución de problemas sociales, el desarrollo nacional, y la formación 
de profesionales y ciudadanos identificados con su realidad (Cevallos, 2014, p. 3). Del mismo modo, también se señalan algunos criterios a tomarse en cuenta para la medición de la calidad docente, como el buen trato en la interacción docente-estudiante, el dominio temático de la materia que imparte, el método de enseñanza, la puntualidad y la asistencia, y la manera de evaluar los aprendizajes (García y Shej, 2014).

Por otro lado, es importante que los profesores desarrollen competencias basadas en la atención a la diversidad, a fin de ofrecer una formación adecuada a las necesidades de sus estudiantes (Díez y Sánchez, 2015). Un docente de calidad es aquel que logra un aprendizaje profundo por parte de los estudiantes, y alcanza las metas establecidas que corresponden, demuestra manejo de contenidos y técnicas metodológicas, despierta el interés por el aprendizaje en sus estudiantes, y respeta sus estilos de aprendizaje (Guzmán, 2011).

En esta línea, Castilla (2011) aporta a la comprensión de la calidad docente, seńalando que se refiere a la necesidad de que el profesorado esté satisfecho e implicado en el aprendizaje de sus alumnos, preocupándose por el logro de aprendizajes, pero a la vez, estar sujeto a evaluaciones externas que acrediten tales competencias. El docente debe estar y expresar conformidad y satisfacción por el servicio educativo que presta (dimensión ética).

Encontramos algunos elementos comunes que se toman en cuenta en la definición de calidad del docente universitario: dimensión temática, dimensión metodológica, dimensión científica, y dimensión ética. Ello concuerda con la Conferencia Mundial sobre la Educación Superior de 2009: la nueva dinámica de la educación superior y la investigación para el cambio social y el desarrollo, que considera como funciones primordiales de la educación superior, las siguientes: investigación, enseñanza, y principios éticos (Unesco, 2009).

\subsection{La calidad educativa en la legislación peruana}

En la legislación peruana, la calidad educativa figura como un eje transversal. La ley 30220 (2014) considera que el último objetivo de la universidad es garantizar y concretizar la inserción laboral de sus egresados, contribuyendo así a una mejora de la calidad de vida de la sociedad humana en su conjunto. Esta inserción laboral figura como el propósito, el fin último de la formación profesional y, a la vez, se convierte en el resultado esperado del servicio educativo superior universitario.

La misma Ley incorpora la variable "calidad» en el servicio educativo superior universitario desde el artículo 5, la cual señala como uno de sus principios no solo la «calidad académica», sino el «mejoramiento continuo» de esta, 
hasta el artículo 109 donde leemos lo siguiente: «La universidad y los colegios profesionales deben mantener una actitud vigilante en cuanto a la calidad del ejercicio profesional de sus afiliados, y deben establecer mecanismos orientados a supervisar y promover el ejercicio eficiente de su profesión». Se compromete a los colegios profesionales en el proceso de vigilancia y promoción del ejercicio eficiente de la profesión; dentro de ella, el ejercicio docente o el servicio educativo superior universitario.

El artículo 6, fines de la universidad (Ley 30220, 2014), declara como uno de sus fines "formar profesionales de alta calidad de manera integral y con pleno sentido de responsabilidad social de acuerdo a las necesidades del país». En consecuencia, deducimos que la formación de profesionales de alta calidad amerita la participación de docentes de alta calidad: «Docentes de alta calidad forman profesionales de alta calidad», con el objetivo primordial de atender las necesidades del país, solucionar sus problemas, liderar el camino hacia el desarrollo social.

Por otro lado, en el artículo 28 de la misma ley, se precisa la necesidad de cumplir con ciertas condiciones básicas de calidad para el otorgamiento del licenciamiento (o autorización de funcionamiento) a una universidad; complementándose con la acreditación de la calidad educativa en el ámbito universitario.

Sucedió algo similar con la educación básica regular. Se publicó la Ley General de Educación (ley 28044, 2003), que en su artículo 13 describe ocho criterios para la medición de la calidad educativa en el nivel básico: (1) lineamientos generales, (2) currículos básicos, (3) inversión mínima por alumno, (4) idoneidad docente, (5) carrera pública docente y administrativa, (6) infraestructura y equipamiento, (7) investigación e innovación educativas, y (8) organización institucional.

En la educación superior universitaria, once años más tarde, con la aprobación de la Política de Aseguramiento de la Calidad de la Educación Superior Universitaria (decreto supremo 016-2015-MINEDU, 2015), se define la calidad como «el grado de ajuste entre las acciones que una universidad, programa académico o carrera llevada a cabo para implementar las orientaciones contenidas en su misión y propósito institucional y los resultados que de estas acciones consigue» (Orozco, 2015, citado por Sunedu, 2015), constituyendo, por primera vez, una definición regulada por ley de la calidad educativa universitaria en el país.

Entendemos, que:

La variable clave en esta definición está constituida por el denominado 'propósito institucional'. ¿Y qué es el propósito institucional? es «el compromiso 
formal que establece la universidad con el conocimiento, el desarrollo del país y la formación integral de los estudiantes». Es decir, todo lo que se propone la Universidad debe estar vinculado con esos 03 elementos. Debe lograr lo que se propone a favor del (1) conocimiento, (2) el desarrollo del país y (3) la formación integral de los estudiantes. Si lo hace, puede sostenerse que es un servicio educativo superior universitario de calidad, por lo tanto, amerita un reconocimiento del Estado y la sociedad (Montenegro, 2016, párrafo 4).

Resulta coherente encajar las cuatro dimensiones del docente universitario que hemos señalado anteriormente, con los tres elementos de la última definición de calidad citada: (1) conocimiento que sería la dimensión temática; (2) formación integral de los estudiantes que sería la dimensión metodológica; y (3) desarrollo del país que incluiría a la dimensión científica y a la dimensión ética.

Unos meses después, se aprueba el «Modelo de Licenciamiento y su implementación en el Sistema Universitario Peruano», el cual incluye las denominadas condiciones básicas de calidad (CBC) que son ocho: (1) objetivos académicos, (2) oferta educativa, (3) infraestructura y equipamiento, (4) líneas de investigación, (5) docentes calificados, (6) servicios educacionales complementarios, (7) mecanismo de mediación e inserción laboral, y (8) transparencia (resolución del Consejo Directivo 006-2015-SUNEDU/CD, 2015), lo que concuerda, y fue delineado con anterioridad, en el artículo 28 de la Ley Universitaria (Ley 30220, 2014).

Tener en cuenta que las $\mathrm{CBC}$ están destinadas a medir el denominado servicio educativo superior universitario, el cual es el servicio educativo que «imparten las instituciones [universitarias], tanto públicas como privadas», sin perder de vista que «el ámbito universitario [es] uno de los puntos de encuentro entre la educación y el mercado laboral y sectores productivos del país» (decreto supremo 016-2015-MINEDU, 2015). Nuevamente, se enfatiza el propósito institucional de atender las demandas de la sociedad, propósito institucional que guiará todo el proceso de la formación profesional.

Encontramos tres similitudes entre los criterios de la calidad educativa del nivel básico con el nivel superior universitario: calidad docente, infraestructura e investigación. A propósito, es la investigación la que se convierte en una actividad fundamental de la universidad peruana actual: «La investigación constituye una función esencial y obligatoria de la universidad, que la fomenta y realiza respondiendo a través de la producción de conocimiento y desarrollo de tecnologías a las necesidades de la sociedad, con especial énfasis en la realidad nacional» (Ley 30220). 
En este contexto, «el problema central de las universidades latinoamericanas es la calidad educativa» (Cevallos, 2014, p. 4), porque quizá «las universidades necesitan dotarse de un sistema de indicadores que permita valorar la calidad de sus docentes, que ayuden a una toma de decisiones informada para su contratación y promoción» (Cruz y Romero-López, 2010, p. 83).

Resumiendo, la calidad es una variable difícil de medir, porque implica la manifestación de la subjetividad de las personas, en tanto, ellas son las autoridades en determinar el grado de satisfacción sentido o alcanzado al consumir determinado bien o servicio. Calidad, como producto o resultado de la percepción de los consumidores, en un determinado contexto.

En consecuencia, la variable calidad trasladada a la docencia universitaria para que ella pueda ser calificada de calidad, demanda del concurso de los receptores de dicho servicio, los estudiantes; obviamente, ubicados en un determinado espacio y tiempo. Estos estudiantes, quienes egresaron de las instituciones educativas y están desempeńándose laboralmente, tienen la autoridad para determinar si el servicio educativo que recibieron fue o no de calidad. En palabras de Álvarez de Zayas (2005), lo harán cuando enfrentados a las exigencias cotidianas del encargo social concluyan si están o no en capacidad de atender, satisfactoriamente, dichas exigencias. Esto se especifica en la denominación de calidad de la educación superior universitaria (MINEDU, 2015), la coherencia o consistencia entre los propósitos institucionales, y los resultados obtenidos.

\section{Metodología}

El diseño del estudio ha sido exploratorio y descriptivo, de corte transversal, así como de tipo fenomenológico hermenéutico, a fin de poder analizar la información obtenida por los ítems no estructurados del instrumento aplicado, utilizando la metodología de la teoría fundamentada de Strauss y Corbin (2002).

La muestra estuvo constituida por 164 estudiantes de tres universidades: Universidad Señor de Sipán (110 de la Escuela Profesional de Psicología), Universidad Privada Juan Mejía Baca (35 de la carrera profesional de Administración Hotelera), y Universidad Nacional Pedro Ruiz Gallo (19 de la Escuela Profesional de Educación), ubicadas en Lambayeque (Perú).

La técnica de recolección de la información fue la encuesta. El instrumento ha sido elaborado por el autor, considerando que no se halló uno que satisfaga el objetivo de la investigación, y luego fue sometido a la validación por juicio de expertos. Fue una encuesta autoaplicada, compuesta por 31 ítems; 
29 estructurados y dos no estructurados. Se ha sintetizado los ocho criterios de calidad de la Educación Básica Regular (Ley 28044, 2003), las ocho condiciones básicas de calidad de la educación universitaria (Ley 30220, 2014), un cuestionario de evaluación de la calidad docente universitaria consensuado entre alumnos y profesores, propuesto por un docente de la Universitat de les Illes Balears (Casero, 2008), y otro cuestionario propuesto por un grupo de docentes de la Universidad de León (Cantón, Valle y Arias, 2008), ambas investigaciones realizadas en España.

La selección y adecuación de los ítems, de los cuestionarios indicados, para la construcción del instrumento del presente estudio, se han realizado considerando el objetivo del estudio. Se han considerado, asimismo, los criterios y condiciones de las leyes indicadas, a fin de facilitar la contrastación de noción de calidad, construida a partir de la percepción de los estudiantes, con la que sugieren dichas normas.

La aplicación del instrumento se realizó en las respectivas aulas de cada una de las universidades a las que pertenecen los estudiantes participantes, entre los meses de julio y agosto de 2017.

El procesamiento y análisis de la información, considerando que el instrumento aplicado contiene ítems estructurados y no estructurados, se ha realizado empleando el método cuantitativo y el método cualitativo. Para el procesamiento de los ítems estructurados, se ha utilizado la versión 24 del programa SPSS (Statistical Package for the Social Sciences); y para el procesamiento y análisis de los ítems no estructurados o abiertos, se ha empleado el programa Atlas.ti versión 7.

En el caso de los ítems no estructurados, considerando la teoría fundamentada de Strauss y Corbin (2002), se crearon diecinueve códigos; dieciocho de los cuales, agrupados en tres familias de códigos, a excepción de uno, por no estar asociado ni depender directamente ni ser causa de ninguno de los otros, como se indica:

Tabla 1. Códigos y familias de la información analizada con Atlas.ti 7

\begin{tabular}{cll}
\hline $\mathrm{N}^{\circ}$ & \multicolumn{1}{c}{ CÓDIGO } & FAMILIA \\
\hline $\mathbf{1}$ & Clases teóricas y prácticas & DIDÁCTICA \\
$\mathbf{2}$ & Comprensivo, amable, amigable, paciente & DIDÁCTICA \\
$\mathbf{3}$ & Dejarse entender, comunicativo, preciso & DIDÁCTICA \\
$\mathbf{4}$ & Didáctico, clases dinámicas, innovador & DIDÁCTICA \\
5 & Escucha, entiende, atienda inquietudes & DIDÁCTICA \\
$\mathbf{6}$ & Exigente & DIDÁCTICA \\
7 & Orienta, ayuda, preocupado por estudiantes & DIDÁCTICA \\
\hline
\end{tabular}




\begin{tabular}{clc}
\hline$N^{\circ}$ & \multicolumn{1}{c}{ CÓDIGO } & FAMILIA \\
\hline $\mathbf{8}$ & Actualizado, capacitado, preparado & DOMINIO TEMÁTICO \\
9 & Dominio temático & DOMINIO TEMÁTICO \\
10 & Investigador & DOMINIO TEMÁTICO \\
11 & Posgrado, magíster, doctor & DOMINIO TEMÁTICO \\
12 & Solucionar problemas & DOMINIO TEMÁTICO \\
13 & Comprometido. Vocación, sabe enseñar & ÉTICA \\
14 & Ético, practique valores, dar ejemplo & ÉTICA \\
15 & Honesto, sincero & ÉTICA \\
16 & Puntual & ÉTICA \\
17 & Respetuoso & ÉTICA \\
18 & Responsable & ÉTICA \\
19 & Vestir formalmente & \\
\hline
\end{tabular}

Fuente: Elaboración propia. Nota: Los códigos y familias han sido construidos para el análisis de la información mediante elaboración de redes, con el programa Atlas.ti 7.

Los códigos han sido agrupados en familias, considerando los enlaces «es parte de» y «está asociado con», proporcionados por el Atlas.ti 7, generando las respectivas redes hermenéuticas para el posterior análisis e interpretación, con el objetivo de construir una noción de calidad de la docencia universitaria a partir de la percepción proporcionada por la muestra de estudio, y luego equipararla con la teorías de la literatura consultada y con las nociones contenidas en la normatividad vigente en el país.

\section{Resultados}

Los resultados lo presentamos en dos partes. La primera parte está relacionada a los ítems estructurados, utilizando un procesamiento cuantitativo de la información, procesada mediante el programa SPSS versión 24; y la segunda se refiere a los ítems no estructurados, utilizando un procesamiento cualitativo de la información, procesada mediante el programa Atlas.ti versión 7.

\subsection{Sobre los ítems estructurados}

El mayor porcentaje de la población que ha participado en la investigación $(90 \%)$ concuerda en que una universidad de calidad, necesariamente, debe contar con docentes de calidad; es decir, una universidad de calidad es inconcebible si no cuenta con docentes de calidad. La calidad del docente universitario, es la que otorga o transfiere calidad al servicio educativo superior universitario: A la universidad donde labora o presta el servicio docente. 
Similar porcentaje de los estudiantes participantes (90\%) indica que este docente de calidad debe elaborar sus propios materiales y recursos didácticos, como diapositivas, esquemas gráficos, textos, artículos, entre otros. No aceptan o consideran apropiado que un docente muestre en aula o trabaje en las sesiones de aprendizaje con materiales y recursos didácticos de otros autores. Esto corresponde a la dimensión metodológica.

En esta misma dimensión, con respecto a que un docente universitario debe emplear en sus sesiones de aprendizaje las redes sociales como Facebook, Twitter, Instagram, LinkedIn, Pinterest, entre otras; la mayoría (el 44\%) indica que sí; frente a un menor porcentaje (13\%) que seńala que depende de los estudiantes, contrastando con un $15 \%$ que opina que no es importante. El uso de las redes sociales debe formar parte de la práctica docente en las sesiones de aprendizaje.

Figura 1. Criterios para evaluar la calidad de la docencia universitaria

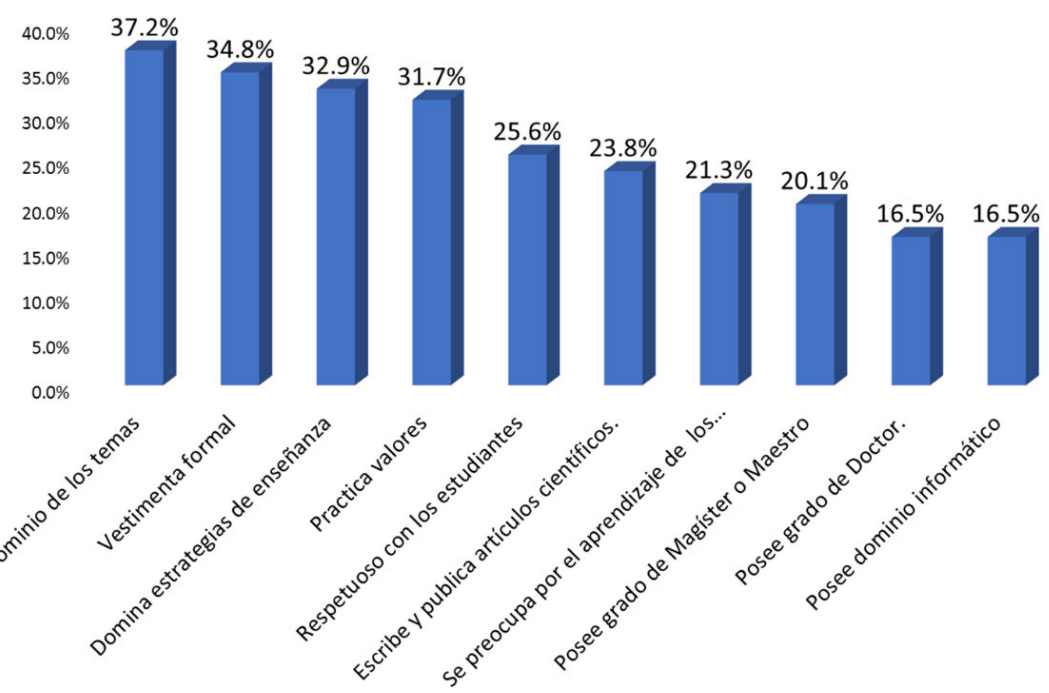

Fuente: Elaboración propia, a partir de la información obtenida con la aplicación del instrumento, y procesada con el programa SPSS versión 24.

Respecto a si en las sesiones de aprendizaje de un docente universitario debería predominar la teoría o la práctica, la mayoría (el 74\%) señala que deberían complementarse ambas modalidades, en un 50\% cada una: mitad teoría, mitad práctica. Sumándose a este grupo, un 15\%, prefiere que las clases sean $100 \%$ prácticas: Ni solo teoría, ni solo práctica. Teórico-prácticas deben ser las sesiones de aprendizaje de un docente universitario de calidad. 
Tener en cuenta que la parte teórica, en una sesión de aprendizaje, refiere al desarrollo temático; es decir, corresponde a la dimensión temática.

Sobre la sostenibilidad del proceso evaluativo de un docente universitario, la mayoría (52\%) es de la idea que debería ser mensualmente; mientras que un menor porcentaje (29\%) concuerda en señalar que la evaluación sea semestralmente. Un docente universitario de calidad debe estar sometido permanentemente a procesos evaluativos de su práctica docente.

No se discute que el docente es el gestor del proceso enseńanza aprendizaje; no obstante, siendo el estudiante el receptor de dicho servicio, a él se le ha otorgado el derecho de participar en la evaluación permanente del docente el cual debería ser, mínimo, una vez en cada semestre académico (Ley 30220). Obviamente, el estudiante representa a la sociedad a la cual, la universidad, debe atender satisfactoriamente, logrando la inserción laboral de sus egresados.

¿Y quiénes deben participar en la evaluación de un docente universitario? La mayoría de las respuestas indica que deben hacerlo las autoridades universitarias (76\%), seguido de los estudiantes (68\%). Muy bajo porcentaje de respuestas concordaron con la participación de otros docentes, egresados y padres de familia (19\%, 18\% y 12\%, respectivamente). Los estudiantes no aceptan que, en la evaluación de los docentes, participen otros docentes, los egresados, o los padres de familia.

Figura 2. Criterios para evaluar la calidad de una universidad

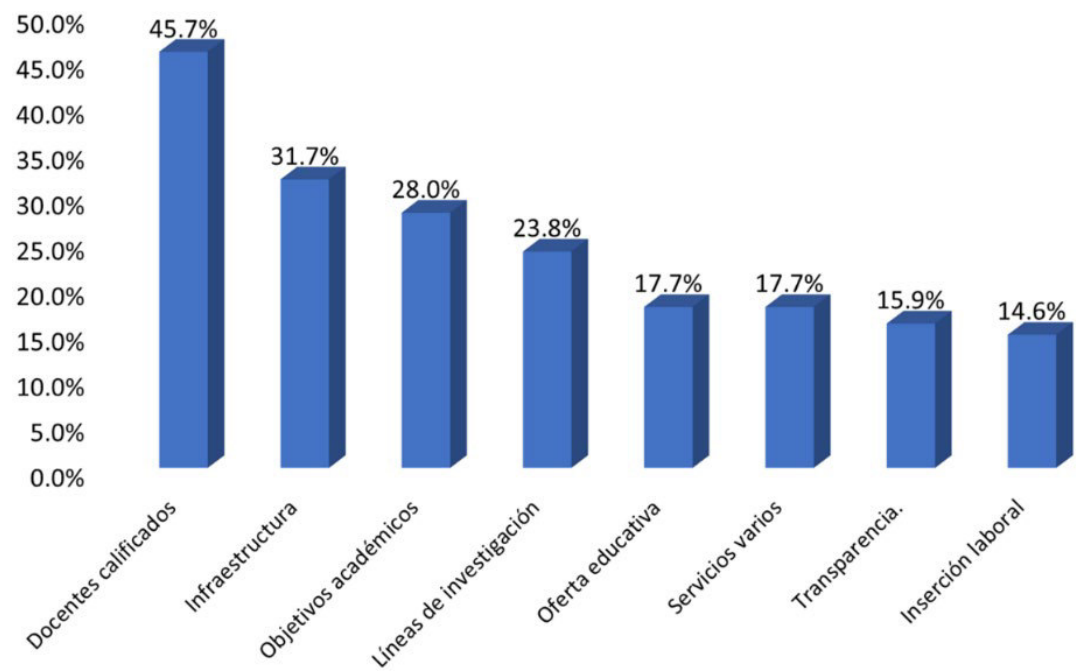

Fuente: Elaboración propia, a partir de la información obtenida con la aplicación del instrumento, y procesada con el programa SPSS versión 24. 
Ello concuerda con las respuestas dadas sobre la priorización de los criterios para medir la calidad universitaria o la calidad del servicio educativo superior universitario. El criterio priorizado para medir la calidad universitaria es el personal docente calificado (46\%), seguido de la Infraestructura y equipamiento adecuado (32\%), la existencia de objetivos académicos, grados y títulos a otorgar, y planes de estudio correspondientes (28\%) y, en cuarto lugar, el $24 \%$ de los estudiantes considera prioritario el criterio Líneas de investigación (correspondiente a la dimensión científica). Otros cuatro criterios: oferta educativa compatible con sus fines propuestos y planeados; servicio médico, social, psicopedagógico, deportivo, entre otros; transparencia (dimensión ética); y, existencia de mecanismos de mediación e inserción laboral obtuvieron una priorización por debajo del 18\%, cada una de ellos.

Y sobre la priorización de los criterios de medición de la calidad docente universitaria; es decir, el referido a la calidad del docente universitario, a la práctica pedagógica en sí, recibió el mayor porcentaje de las respuestas, el criterio dominio de los temas, materias o asignaturas trabajados en el aula (referido a la dimensión temática) con un 37\%; en segundo lugar, la vestimenta formal (terno, traje en las damas, camisa y corbata, vestir elegante, etc.) con un $45 \%$; desplazando al tercer lugar al criterio Domina estrategias de enseñanza (se deja entender, es preciso y organizado; correspondiente a la dimensión metodológica) con un 33\% de las respuestas; ocupando el cuarto lugar de priorización el criterio práctica de valores, como la puntualidad, responsabilidad, honestidad, sinceridad, entre otros (correspondiente a la dimensión ética).

Otros seis criterios considerados recibieron un porcentaje de respuestas de $25 \%$ a menos. (Respetuoso con los estudiantes. Escucha atentamente, es paciente, etc.; posee grado de magíster o Maestro, o cualquier otro grado de similar nivel; posee grado de doctor; se preocupa por el aprendizaje de los estudiantes, aclara las dudas; posee dominio informático: computadoras, redes sociales, programas educativos, etc.; y, escribe y publica artículos científicos).

\subsection{Sobre los ítems no estructurados}

Se utilizó el programa Atlas.ti versión 7. Se construyeron tres grandes redes de códigos, pertenecientes a las tres familias de códigos creadas, las cuales agrupan a los 18 de los 19 códigos hermenéuticos elaborados para la interpretación de la información (Tabla 1). 
Tabla 1. Códigos y familias de la información analizada con Atlas.ti 7

\begin{tabular}{clc}
\hline $\mathrm{N}^{\circ}$ & \multicolumn{1}{c}{ CÓDIGO } & FAMILIA \\
\hline 1 & Clases teóricas y prácticas & DIDÁCTICA \\
2 & Comprensivo, amable, amigable, paciente & DIDÁCTICA \\
3 & Dejarse entender, comunicativo, preciso & DIDÁCTICA \\
4 & Didáctico, clases dinámicas, innovador & DIDÁCTICA \\
5 & Escucha, entiende, atienda inquietudes & DIDÁCTICA \\
6 & Exigente & DIDÁCTICA \\
7 & Orienta, ayuda, preocupado por estudiantes & DIDÁCTICA \\
8 & Actualizado, capacitado, preparado & DOMINIO TEMÁTICO \\
9 & Dominio temático & DOMINIO TEMÁTICO \\
10 & Investigador & DOMINIO TEMÁTICO \\
11 & Posgrado, magíster, doctor & DOMINIO TEMÁTICO \\
12 & Solucionar problemas & DOMINIO TEMÁTICO \\
13 & Comprometido. Vocación, sabe enseñar & ÉTICA \\
14 & Ético, practique valores, dar ejemplo & ÉTICA \\
15 & Honesto, sincero & ÉTICA \\
16 & Puntual & ÉTICA \\
17 & Respetuoso & ÉTICA \\
18 & Responsable & ÉTICA \\
19 & Vestir formalmente & \\
\hline
\end{tabular}

Fuente: Elaboración propia. Nota: Los códigos y familias, han sido construidos para el análisis de la información mediante elaboración de redes, con el programa Atlas.ti 7.

Los códigos con mayores frecuencias en la información proporcionada por los estudiantes (Tabla 2), y a la vez, con mayores vínculos y asociaciones en las redes de familias, contienen sus respectivos memos a fin de precisar sus significados y facilitar la interpretación de la información, antes de la creación de las redes hermenéuticas de las familias de códigos.

Tabla 2. Frecuencia de los códigos, y familia de pertenencia

\begin{tabular}{|c|c|c|c|}
\hline $\mathrm{N}^{\circ}$ & CÓDIGO & FAMILIA & FRECUENCIA \\
\hline 1 & $\begin{array}{l}\text { Didáctico, clases dinámicas, } \\
\text { innovador }\end{array}$ & DIDÁCTICA & 68 \\
\hline 2 & Dominio temático & DOMINIO TEMÁTICO & 55 \\
\hline 3 & $\begin{array}{l}\text { Dejarse entender, comunicativo, } \\
\text { preciso }\end{array}$ & DIDÁCTICA & 38 \\
\hline 4 & $\begin{array}{l}\text { Actualizado, capacitado, } \\
\text { preparado }\end{array}$ & DOMINIO TEMÁTICO & 35 \\
\hline
\end{tabular}




\begin{tabular}{|c|c|c|c|}
\hline $\mathrm{N}^{\circ}$ & CÓDIGO & FAMILIA & FRECUENCIA \\
\hline 5 & $\begin{array}{l}\text { Ético, practique valores, dar } \\
\text { ejemplo }\end{array}$ & ÉTICA & 34 \\
\hline 6 & $\begin{array}{l}\text { Comprensivo, amable, } \\
\text { amigable, paciente }\end{array}$ & DIDÁCTICA & 32 \\
\hline 7 & Respetuoso & ÉTICA & 30 \\
\hline 8 & Responsable & ÉTICA & 29 \\
\hline 9 & $\begin{array}{l}\text { Orienta, ayuda, preocupado por } \\
\text { estudiantes }\end{array}$ & DIDÁCTICA & 27 \\
\hline 10 & Puntual & ÉTICA & 23 \\
\hline 11 & Clases teóricas y prácticas & DIDÁCTICA & 19 \\
\hline 12 & $\begin{array}{l}\text { Escucha, entiende, atienda } \\
\text { inquietudes }\end{array}$ & DIDÁCTICA & 17 \\
\hline 13 & $\begin{array}{l}\text { Comprometido. Vocación, sabe } \\
\text { enseñar }\end{array}$ & ÉTICA & 15 \\
\hline 14 & Posgrado, magíster, doctor & DOMINIO TEMÁTICO & 15 \\
\hline 15 & Vestir formalmente* & & 10 \\
\hline 16 & $\begin{array}{l}\text { Exigente. Promueve } \\
\text { responsabilidad académica en el } \\
\text { estudiante }\end{array}$ & DIDÁCTICA & 5 \\
\hline 17 & Investigador & DOMINIO TEMÁTICO & 5 \\
\hline 18 & Honesto, sincero & ÉTICA & 5 \\
\hline 19 & Solucionar problemas & DOMINIO TEMÁTICO & 2 \\
\hline
\end{tabular}

Fuente: Elaboración propia, a partir de la información obtenida con la aplicación del instrumento. Nota: Las frecuencias son las veces pronunciadas por los estudiantes. Es el número de repeticiones operadas con el programa Atlas.ti 7.

* El código Vestir formalmente, al no ser parte de ningún otro código, ni estar asociado directamente, no fue considerado en ninguna de las tres familias de códigos, creadas.

Los memos construidos también corresponden a las cuatro dimensiones que hemos identificado en la literatura consultada, sobre la calidad del docente universitario: dimensión temática, dimensión metodológica, dimensión científica y dimensión ética. A continuación, el detalle de los mismos presentados según orden descendente de las frecuencias respectivas.

Didáctico, clases dinámicas, innovador (68). Constituye un conjunto universal, para señalar que un docente universitario de calidad debe conocer, dominar y aplicar estrategias que faciliten el aprendizaje, al mismo tiempo que convierte el ambiente en el aula, en un espacio saludable, recreativo que cautive y mantenga la atención del estudiante; que emplee medios y materiales de última tecnología, programas informáticos, redes sociales, diapositivas, videos, entre otros. Al mismo tiempo, indican que su metodología debe ser innovadora. 
Siempre relacionan lo didáctico con clases dinámicas, innovadoras, recreativas o divertidas, como algunos lo han señalado. Ninguno ha señalado o ha vinculado el ser didáctico con dominio del tema. Dominar el tema es una categoría o característica independiente (Figura 1).

Dominio temático (55). A este código se adhieren la capacitación permanente y preparación anticipada de la clase. Conocer el tema de clase. Además, señalan que debe tener la especialidad de la asignatura, e incluso, estudios de posgrado. Este dominio temático implica que el docente atienda las dudas e inquietudes de los estudiantes, responda a las preguntas, sea claro y preciso, dejarse entender; pero a la vez, sea capaz de solucionar los problemas que se presentan en el aula. Todo ello evidencia su dominio del tema o asignatura a su cargo. Además, dominar la teoría y la práctica (Figura 2).

Dejarse entender, ser comunicativo y preciso (38). Como el docente es una persona preparada, conoce su tema, maneja didáctica, tiene experiencia y formación especializada, y el estudiante, no; entonces, el docente debe tener la capacidad de dejarse entender, ser claro, preciso, disponible ante las dudas e inquietudes de los estudiantes. El docente siempre debe comunicarse con el estudiante, con respeto y amabilidad. Ser comprensivo y entenderlo; dominar su tema, pero dejarse entender; dominar estrategias de enseñanza, pero ser divertido.

Actualizado, capacitado, preparado (35). Lo relacionan con estudios de posgrado, y otras especializaciones sobre su asignatura o temas que desarrolla en el aula. Esto se evidenciará en el dominio de los temas, en el manejo de información actualizada, información nueva, que deberá compartir con los estudiantes.

Ético, practique valores, dar ejemplo (34). Se trata no solo que el docente universitario practique valores, sino que evidencia con su comportamiento; como lo dicen, que dé el ejemplo. Asimismo, que cumpla valores, pero, a la vez, que inculque, motive, promueva y exija que el estudiante también sea ético. Dentro de los valores, algunos han sido señalados directamente, por ejemplo: responsabilidad, respeto, puntualidad, compromiso, honestidad, sinceridad; en este orden de prioridad, o frecuencia en las respuestas. No han sido mencionadas directamente otras categorías que podrían ser considerados también valores; como, por ejemplo: el estudio, el trabajo, el aprendizaje, la verdad, la amistad, la autoestima, entre otros (Figura 3).

Comprensivo, amable, amigable, paciente (32). El docente debe comprender al estudiante, cuando tiene problemas de aprendizaje o dificultades para alcanzar los aprendizajes esperados. El docente debe ser amable, 
amigable, tener paciencia, porque es una persona preparada, conoce metodología de enseñanza. Todo ello, en un ambiente de respeto y confianza. En suma, indican que no debe tratar mal al estudiante que no aprende o no entiende fácilmente un tema explicado por el docente. Sin embargo, también el docente, debe dejarse entender.

Respetuoso (30). Básicamente, respeto. Alude un buen trato al estudiante. Por ejemplo, en situaciones de problemas de aprendizaje o dificultades para entender un tema; que lo explique con claridad y con un lenguaje preciso, pero no solo que respete al estudiante, sino, que se haga respetar por él.

Responsable (29). Tiene que ver con asistencia a las clases programadas, que no falte, y si falta, que avise con anticipación. Que cumpla con sus actividades programadas según el sílabo de la asignatura. Pero no solo que asista a sus clases, sino, que sea puntual, y, además, que siempre esté formalmente vestido, como debe ser un docente universitario y un profesional de calidad. La imagen también cuenta.

Orienta, ayuda, preocupado por el estudiante (27). Cuando señalan que un docente de calidad se preocupe por el estudiante, precisan que se preocupe por su aprendizaje. Es decir, que el docente se interese por el aprendizaje del estudiante; y en este proceso de preocupación que lo oriente y ayuda a lograr dichos aprendizajes, cuando evidencia dificultades o limitaciones para entender los temas trabajados en la asignatura o en el aula de clases. En varias respuestas que indican esta preocupación del docente, lo relacionan con la exigencia. El docente debe ser exigente, pero si esta exigencia revela dificultades de aprendizaje, el docente debe ayudar, orientar, asistir al estudiante para superar dichas dificultades.

Clases teóricas y prácticas (19). El docente universitario de calidad no solo debe decir cosas nuevas, sino hacerlas; es decir, poner en práctica lo que enseña teóricamente, porque haciendo cosas se aprende más, indican los estudiantes.

Exigente. Promueve responsabilidad académica en el estudiante (5). Cuando indican que un docente universitario de calidad debe ser exigente, se refieren a que debe monitorear o seguir el comportamiento académico del estudiante, que cumpla con las actividades programadas; es decir, que sea responsable al igual que el docente.

Los diagramas de las redes de las familias de códigos o nodos, diseñados con el programa Atlas.ti 7, se presentan a continuación: 


\section{Figura 3. Red de la familia de códigos Didáctica}

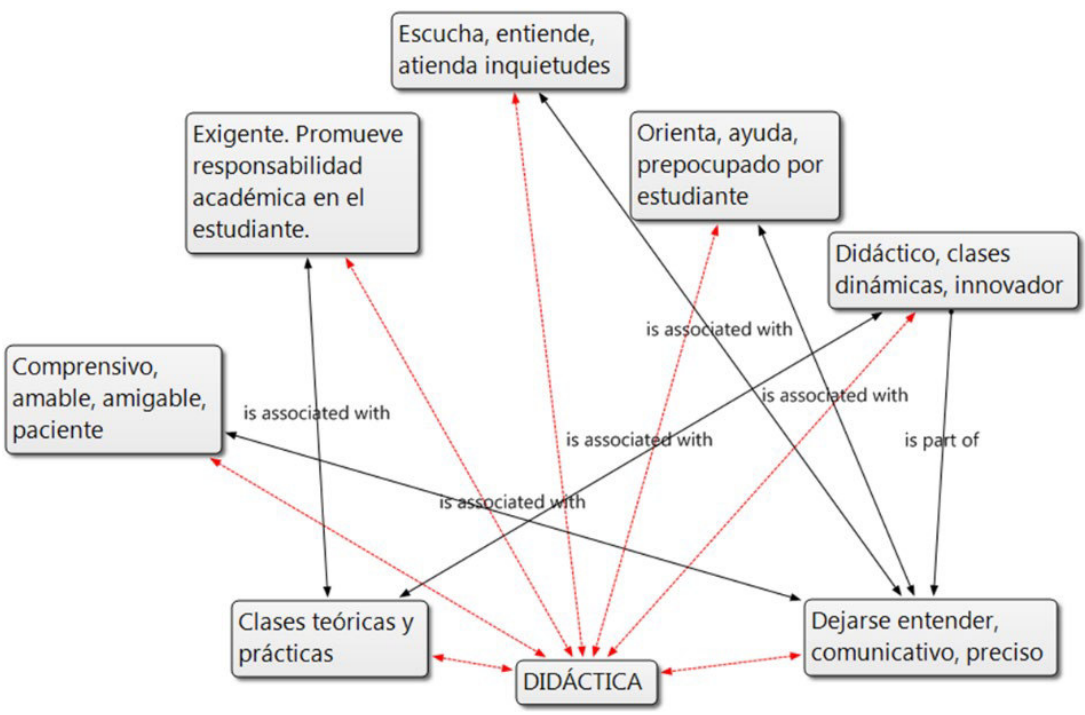

Fuente: Elaboración propia. Nota: Elaborado con el programa Atlas.ti 7, a partir de la información obtenida con la aplicación del instrumento.

\section{Figura 4. Red de la familia de códigos Dominio temático}

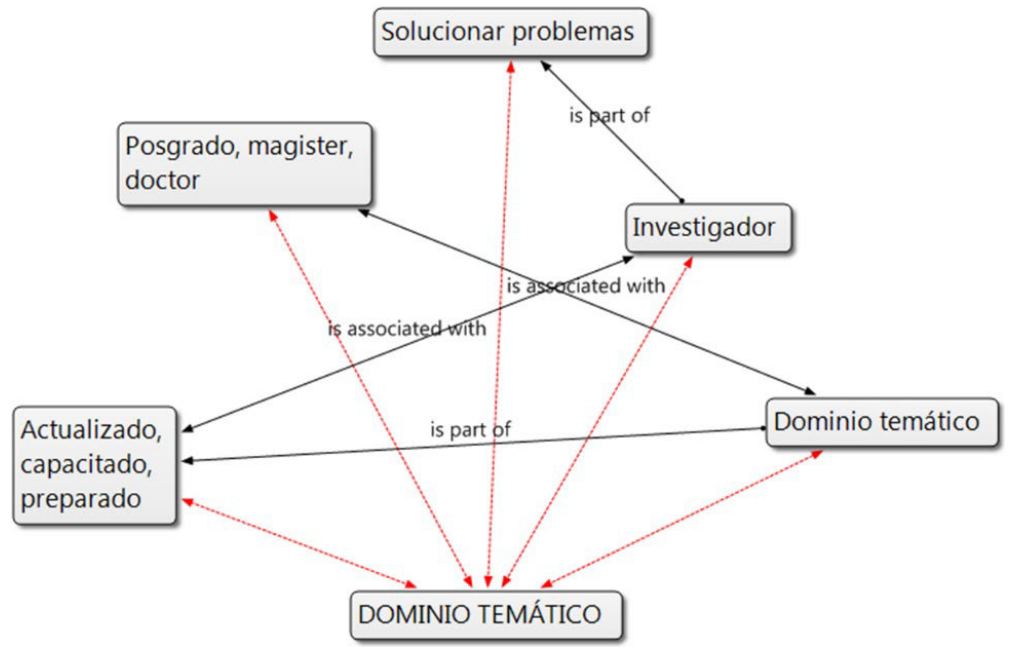

Fuente: Elaboración propia. Nota: Elaborado con el programa Atlas.ti 7, a partir de la información obtenida con la aplicación del instrumento. 
Figura 5. Red de la familia de códigos Ética

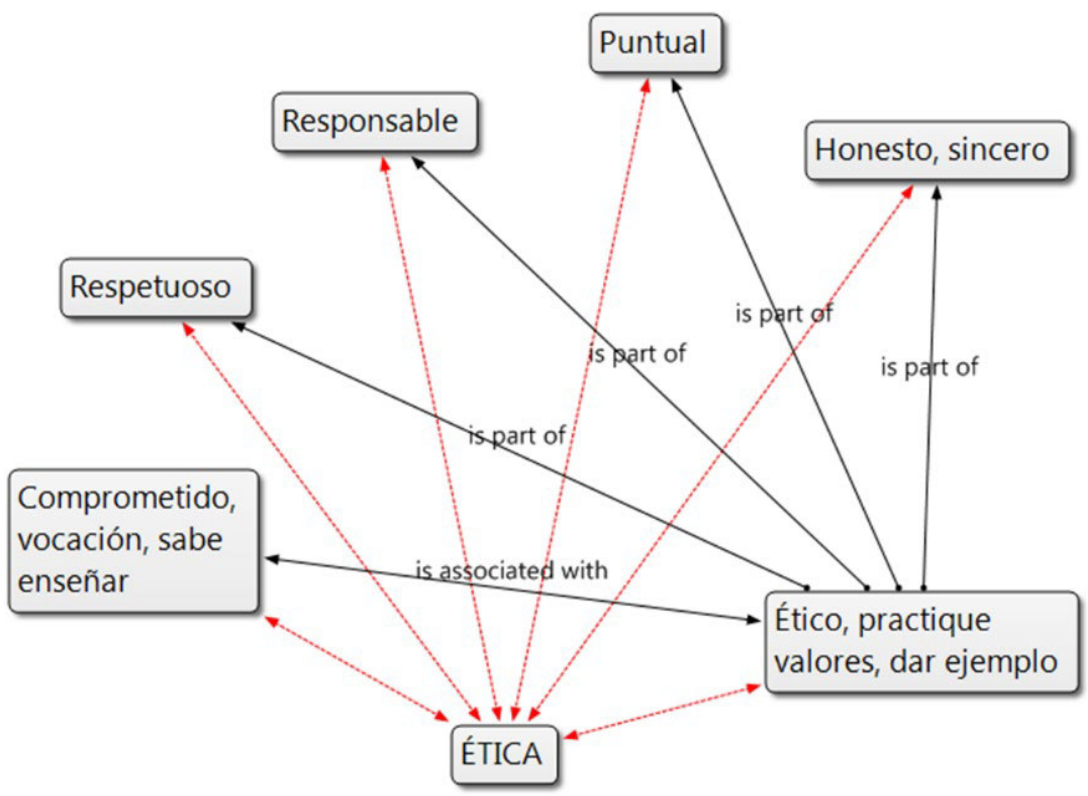

Fuente: Elaboración propia. Nota: Elaborado con el programa Atlas.ti 7, a partir de la información obtenida con la aplicación del instrumento.

\section{Discusión}

La literatura consultada nos indica que son cuatro las dimensiones a tomar en cuenta para evaluar la calidad de la docencia universitaria:

- La dimensión temática; dominio teórico acorde a la especialidad del docente.

- La dimensión metodológica; relativa a la didáctica que asegure el logro de nuevos aprendizajes en los estudiantes.

- La dimensión científica; a fin de producir nuevos conocimientos que prometan solucionar la problemática social del entorno.

- La dimensión ética; practicar y enseñar valores como la honestidad, responsabilidad, puntualidad y el buen trato a los estudiantes. 
Todo ello concuerda con las condiciones básicas de calidad, detalladas en el «Modelo de Licenciamiento y su implementación en el Sistema Universitario Peruano» (resolución del Consejo Directivo 006-2015-SUNEDU/CD, 2015), las que han sido trasladadas al instrumento utilizado en la presente investigación. Los resultados obtenidos en el cuestionario aplicado, muestran un orden como se indica en la siguiente tabla:

Tabla 3. Comparación del orden de prioridad de las condiciones básicas de calidad

\begin{tabular}{l|l}
\hline \multicolumn{2}{c}{ CBC } \\
\hline \multicolumn{1}{c}{ Orden en el Modelo de Licenciamiento } & \multicolumn{1}{c}{ Orden priorizado por los estudiantes } \\
\hline $\begin{array}{l}\text { Objetivos académicos, grados y } \\
\text { títulos a otorgar, y planes de estudio } \\
\text { correspondientes. }\end{array}$ & Personal docente calificado \\
$\begin{array}{l}\text { Oferta educativa compatible con sus fines } \\
\text { propuestos y planeados }\end{array}$ & $\begin{array}{l}\text { Infraestructura y equipamiento adecuados } \\
\text { (aulas, bibliotecas, laboratorios, etc.) }\end{array}$ \\
$\begin{array}{l}\text { Infraestructura y equipamiento } \\
\text { adecuados (aulas, bibliotecas, }\end{array}$ & $\begin{array}{l}\text { Objetivos académicos, grados y } \\
\text { títulos a otorgar, y planes de estudio } \\
\text { laboratorios, etc.) }\end{array}$ \\
$\begin{array}{l}\text { Líneas de investigación a ser desarrolladas } \\
\text { Personal docente calificado }\end{array}$ & $\begin{array}{l}\text { Líneas de investigación a ser desarrolladas } \\
\text { Oferta educativa compatible con sus fines } \\
\text { propuestos y planeados }\end{array}$ \\
$\begin{array}{l}\text { Servicios educacionales complementarios: } \\
\text { servicio médico, social, psicopedagógico, } \\
\text { deportivo, entre otros }\end{array}$ & $\begin{array}{l}\text { Servicios educacionales complementarios: } \\
\text { servicio médico, social, psicopedagógico, } \\
\text { deportivo, entre otros }\end{array}$ \\
$\begin{array}{l}\text { Existencia de mecanismos de mediación } \\
\text { e inserción laboral (Bolsa de Trabajo u } \\
\text { otros) }\end{array}$ & $\begin{array}{l}\text { Transparencia } \\
\text { Transparencia }\end{array}$ \\
\hline
\end{tabular}

Elaboración propia, a partir de la información obtenida con la aplicación del instrumento. Nota: CBC es condiciones básicas de calidad, según el «Modelo de Licenciamiento y su implementación en el Sistema Universitario Peruano", aprobado con resolución del Consejo Directivo 006 -2015-SUNEDU/CD, 2015, considerando el artículo 28 de la Ley Universitaria, ley 30220 (2014).

En nuestro caso, se evidencia que el estudiante maneja su concepto de calidad docente, el mismo que es similar a la noción que contiene la legislación vigente en el país, pero también a la que encontramos en la literatura consultada. Además, la calidad docente es inherente a la calidad educativa. 


\subsection{Sobre la calidad educativa}

Las condiciones para medir la calidad del servicio educativo universitario, descritas en la norma respectiva, también son considerados por los estudiantes. Aunque, no necesariamente presentan el mismo orden de prioridad.

Podemos ver que la condición objetivos académicos, grados y títulos a otorgar, y planes de estudio correspondientes, que figura en primer orden en la norma seńalada, es considerada de tercera prioridad por los estudiantes participantes. Por otro lado, mientras que para los estudiantes es de primera prioridad que una universidad de calidad, cuente con personal docente calificado; en la norma descrita, se ubica en el cuarto lugar.

Asimismo, con la condición oferta educativa compatible con sus fines propuestos y planeados, para la norma descrita, es ubicada en segundo lugar; para los estudiantes que participaron en la investigación, es un criterio de medición de la calidad universitaria de quinta prioridad.

No hay mayor discusión con la condición líneas de investigación a ser desarrolladas; en ambos casos, tanto como para la norma descrita como para los estudiantes que participaron en la investigación, es ubicado en el cuarto lugar de prioridad. (Podría resultar poco contradictorio que las Condiciones Básicas de Calidad ubiquen a las Líneas de investigación a ser desarrolladas en cuarto lugar cuando la Ley Universitaria Ley 30220 (2014) considera a la investigación como una función esencial y obligatoria de la universidad, artículo 48. Una universidad que no investiga, no es universidad).

\subsection{Sobre la calidad docente}

Referente a la dimensión metodológica. Los estudiantes participantes respaldan el empleo de las redes sociales como Facebook, Twitter, Instagram, LinkedIn, Pinterest, etc., por parte de los docentes universitarios en las sesiones de aprendizaje, coincide con el estudio de Rebollo et al. (2009) y Stromquist (2009), citados por Buela-Casal y Castro (2011), quienes sostienen que "para el desarrollo de la innovación docente demandada por el EEES (Espacio Europeo de Educación Superior) ha sido muy importante la utilización, cada vez mayor, de las nuevas tecnologías de la información y la comunicación».

Es elemental el dominio de estrategias de enseñanza; concordando con Guzmán (2011), al señalar que «el profesor de educación superior tendrá que volverse más profesional, es decir, tendrá que formalizar su preparación, sobre todo en lo que atańe a la didáctica, y comenzar a impartir clases solo después de demostrar el dominio de las habilidades docentes» (p. 132). 
Del mismo modo, concuerda con Ramsden (citado por Guzmán, 2011), al escribir que de un docente universitario:

Se espera que sea un excelente maestro, que diseñe cursos y aplique métodos de enseñanza adecuados para cumplir con los requerimientos de una población estudiantil heterogénea, que sepa lidiar con grupos numerosos de estudiantes, que utilice apropiadamente las nuevas tecnologías de la información y la comunicación [...]. (p. 132).

Igualmente, en el espacio Europeo de Educación Superior, a fin de mejorar la calidad docente, se está volviendo la mirada hacia «métodos pedagógicos innovadores», con el objetivo de lograr una «repercusión inmediata en el aula» (Castilla, 2011, p. 158).

Referente a la dimensión temática. La calidad de un docente universitario refiere a los estudiantes, también se mide por el dominio de los temas, estar actualizado y atender dudas sobre el desarrollo de los mismos.

Otra de las investigaciones que concuerda con nuestras conclusiones es la de Alvarado et al. (2016), quienes confirman que se aprecia mucho por parte de los estudiantes universitarios «el conocimiento y el cumplimiento de los profesores al impartir cátedra» (p. 61). Concluyen también que, de las cuatro características particulares de la calidad del servicio educativo, está «la combinación de aspectos teóricos y prácticos, y la utilización de medios de enseñanza modernos» (p. 64); en nuestro caso, equivale al uso de las redes sociales en las sesiones de aprendizaje por los docentes universitarios de calidad.

Si bien es cierto, la dimensión temática y la dimensión metodológica pueden verse como ejes centrales en la evaluación de un docente universitario; el ser buena persona, que alude a la práctica de valores, también es predominante (dimensión ética). Por ejemplo, Rizo (1999) acentúa que, si un docente es impuntual, resulta mal evaluado por los estudiantes; de forma similar, si trata mal, no respeta, o no tiene paciencia para enseñar.

La dimensión científica es transversal a las otras tres dimensiones, porque proporciona nueva información (además de actualizada, contextualizada), que orienta y conduce el desarrollo de la dimensión temática y dimensión metodológica, así como de la dimensión ética, acorde a las necesidades y demandas de los estudiantes como miembros de la sociedad a la que debe atender el servicio educativo, proponiendo soluciones a la problemática que presenta.

Obviamente, el estudiante es uno de los agentes participantes en el proceso de evaluación de la calidad docente y así se ha estructurado todo el proceso de medición de las condiciones básicas de calidad (CBC), a fin de conceder la licencia institucional a las universidades públicas y privadas para ofrecer el 
denominado servicio educativo superior universitario (ley 30220). Es ineludible la participación del estudiante en la evaluación docente. Efectivamente, el docente no ha dejado de cumplir su papel de evaluador como gestor de su práctica pedagógica; sin embargo, y en simultáneo, ha pasado a ser, también, objeto de evaluación. Su práctica pedagógica está siendo permanentemente evaluada.

\section{Conclusión}

Una institución educativa universitaria de calidad debe contar con personal docente calificado, tener una infraestructura y equipamiento adecuado, producir conocimiento científico; pero también, practicar la transparencia y proveer servicios educacionales complementarios.

En cuanto a la calidad del docente universitario, tiene que ver con cuatro dimensiones inherentes a su quehacer educativo y práctica pedagógica: la dimensión temática, la dimensión metodológica, la dimensión científica y la dimensión ética.

En lo referente a la dimensión metodológica, la muestra del estudio refiere que un docente de calidad debe elaborar sus propios materiales y recursos didácticos: diapositivas, esquemas gráficos, textos, artículos, entre otros; debe emplear en sus sesiones de aprendizaje las redes sociales como Facebook, Twitter, Instagram, LinkedIn, Pinterest, entre otras; sus sesiones de aprendizaje deben ser teóricas y prácticas, equitativamente distribuidas (50\% teoría y $50 \%$ práctica).

Del mismo modo, tiene que dejarse entender, ser comunicativo y preciso en su lenguaje; debe orientar, ayudar, y preocuparse por el aprendizaje de los estudiantes; tiene que saber escuchar, entender y atender las inquietudes, dudas y otros requerimientos académicos de ellos; debe ser comprensivo, amable, amigable y paciente; todo ello, en un ambiente de exigencia académica que incluya al docente mismo y a los estudiantes.

Sobre la dimensión temática, encontramos que un docente debe mostrarse actualizado, capacitado y preparado para el desarrollo de los temas programados en su asignatura, tener posgrados académicos y tener la capacidad de solucionar los problemas que se presenten en las sesiones de aprendizaje, las mismas que deben ser teóricas y prácticas.

En la dimensión ética, se debe ser una buena persona y un buen ciudadano. Un docente universitario de calidad es didáctico y académico, pero también ético. Asimismo, practica valores y los inculca en sus estudiantes. Es puntual, responsable, respetuoso, honesto y sincero, comprometido con su quehacer docente, que tenga vocación y que sepa enseñar. 
Relativo a la dimensión científica. El docente universitario debe investigar y compartir sus investigaciones con los estudiantes; del mismo modo, los nuevos conocimientos deben revertirse en la mejora de su práctica pedagógica, permanentemente. Obviamente, la investigación científica puede percibirse como el cimiento de toda la estructura de la docencia universitaria. Se investiga para repotenciar la dimensión temática (dominio y actualización permanente de los temas desarrollados), la dimensión metodológica (dominio y actualización permanente de la didáctica universitaria) y la dimensión ética (transparencia en el discurso y en la acción. El docente universitario debe ser una persona íntegra).

Sintetizando, para la muestra del estudio, un docente de calidad deberá poseer dominio en cuatro dimensiones: dimensión temática, dimensión metodológica, dimensión científica (ser un investigador) y dimensión ética. Ello concuerda con las cuatro dimensiones que encontramos en la literatura consultada, asimismo, con la normativa vigente en el país. De forma directa, con dos condiciones para medir la calidad educativa universitaria: personal docente calificado (dimensión temática y dimensión metodológica), y líneas de investigación a ser desarrolladas (dimensión científica); e indirectamente, con otras condiciones: servicios educacionales complementarios y transparencia. Esta transparencia tiene que ver con acceso libre a información veraz y oportuna, de parte del docente, así como la visibilidad de su idoneidad y probidad (dimensión ética).

El docente universitario, si bien es cierto, realiza su labor pedagógica hacia un grupo de estudiantes, su mirada no se aleja de la sociedad que, en esa aula o espacio didáctico, está representada por ese grupo de jóvenes que atiende su discurso, su práctica y su modo de ser (como profesional y como persona), con ansias de concluir preparados y revertir sus aprendizajes en beneficio de la sociedad a la que pertenecen, solucionando su diversidad problemática. Esta correlación entre formación profesional y desarrollo social, entre propósito institucional e impacto social resultante, nos permitirá dilucidar la calidad el servicio educativo superior universitario, en un contexto determinado.

En suma, la noción de un docente universitario de calidad es la siguiente: un profesional que evidencie dominio de su especialidad con temas actualizados (dimensión temática), aplique una didáctica capaz de promover y lograr nuevos aprendizajes en sus estudiantes para el desarrollo social (dimensión metodológica), domine y ejecute la metodología de la investigación científica para nutrir su especialidad permanentemente (dimensión científica) y sea transparente, que practique y enseñe valores que garanticen una convivencia académica saludable a favor de una sociedad humana cada día más sana (dimensión ética). 


\section{REFERENCIAS BIBLIOGRÁFICAS}

Alvarado, E., Ramírez, D. y Téllez, E. (2016). Percepción de la calidad educativa: caso aplicado a estudiantes de la Universidad Autónoma de Nuevo León y del Instituto Tecnológico de Estudios Superiores de Monterrey. Revista de La Educación Superior, 45(180), 55-74. https://doi.org/10.1016/j. resu.2016.06.006

Álvarez de Zayas, C. (2005). Didáctica de la educación superior (sétima edición). La Habana: Fondo Editorial: FACHSE, UNRPG.

Buela-Casal, G. y Castro, Á. (2011). La evaluación de la calidad docente y de la investigación hoy. Cultura y Educación, 23(2), 253-257. https://doi. org/10.1174/113564011795944668

Cantón, I., Valle, R. y Arias, A. (2008). Calidad de la docencia universitaria: procesos clave. Educatio Siglo XXI: Revista de La Facultad de Educación, (26), 121-159. Recuperado de http://dialnet.unirioja.es/servlet/articulo?codig o=2882327\&orden=191563\&info=link\%5Cnhttp://dialnet.unirioja.es/ servlet/extart? codigo $=2882327$

Casero, A. (2008). Propuesta de un cuestionario de evaluación de la calidad docente universitaria consensuado entre alumnos y profesores. Revista de Investigación Educativa, 26(1), 25-44.

Castilla, F. (2011). Calidad docente en el ámbito universitario: un estudio comparativo de las universidades andaluzas. Revista de Educación en Contabilidad, Finanzas y Administración de Empresas, (2). Recuperado de https://dialnet. unirioja.es/servlet/articulo?codigo $=3965502$

Cevallos, D. (2014). La calidad educativa en la realidad universitaria peruana frente al contexto latinoamericano. Flumen, 7(1), 3-8.

Cruz, M. y Romero-López, A. (2010). Indicadores de excelencia docente en la Universidad de Granada. Revista Portuguesa de Pedagogía, 83-117. https:// doi.org/10.14195/1647-8614_44-1_4

Danielson, C. (2007). Enhancing professional practice: A framework for teaching ( $2^{a}$ ed.). Alexandria: VA: Association for Supervision and Curriculum Development.

Darling-Hammond, L. (2012). Creating a comprehensive system for evaluating and supporting effective teaching. Stanford: Stanford University, Stanford Center for Opportunity Policy in Education.

Decreto Supremo 016-2015-MINEDU (2015) Aprueban la Política de Aseguramiento de la Calidad de la Educación Superior Universitaria. Publicado el 26 de setiembre de 2015. Recuperado de https://elperuano. pe/NormasElperuano/2015/09/26/1292708-1.html 
Díez, E. y Sánchez, S. (2015). Diseño universal para el aprendizaje como metodología docente para atender a la diversidad en la universidad. Aula Abierta, 43(2), 87-93. https://doi.org/10.1016/j.aula.2014.12.002

García, J. y Shej, A. (2014). Los criterios que emplean los estudiantes universitarios para evaluar la ineficacia docente de sus profesores. Perfiles Educativos, 36(143), 124-139. https://doi.org/10.1016/S0185-2698(14)70613-0

Guzmán, J. (2011). Perfiles educativos. Perfiles Educativos, 33(SPE), 129-141.

Hanushek, E. y Rivkin, S. (2006). Teacher quality. En E. Hanushek y F. Welch (Eds.), Handbook of the Economics of Education (pp. 1051-1078). Holanda: Elsevier. https://doi.org/10.1016/S1574-0692(06)02018-6

Ley 28044 Ley General de Educación. Publicada el 29 de julio de 2003. Recuperado de http://www.leyes.congreso.gob.pe/Documentos/Leyes/28044.pdf

Ley 30220 Ley Universitaria. Publicada el 09 de julio de 2014. Recuperado de http://www2.congreso.gob.pe/Sicr/TraDocEstProc/Expvirt_2011.nsf/ Repexpvirt?OpenForm \&Db=201100154\&View

Millman, J. (1997). Grading teachers, grading schools: Is student achievement a valid evaluation measure? Thousand Oaks, CA: Corwin Press.

Montenegro, J. (6 de enero de 2016). La calidad en la educación superior universitaria (I) [Artículo en blog]. Recuperado de https://escritosdominicales. blogspot.com/2016/01/la-calidad-es-un-factor-que-esta.html

Montero, L., Trivińo, X., Dois, A., Sirhan, M. y Leiva, L. (2017). Percepción de los académicos del rol docente del médico. Investigación en Educación Médica. https://doi.org/10.1016/j.riem.2017.01.001

Papay, J. (2012). Refocusing the debate: Assessing the purposes and tools of teacher evaluation. Harvard Educational Review, 82, 123-141. https://doi. org/10.17763/haer.82.1.v40p0833345w6384

Resolución del Consejo Directivo 006-2015-SUNEDU/CD. Aprueban el «Modelo de Licenciamiento y su implementación en el Sistema Universitario Peruano». Publicado el 3 de noviembre de 2015. Recuperado de http://repositorio.minedu.gob.pe/bitstream/handle/123456789/4565/ El\%20 Modelo\%20de\%20 Licenciamiento\%20y\%20su \% 20 Implementaci\%C3\%B3n\%20en\%20el\%20Sistema\%20Universitario\% 20Peruano.pdf?sequence $=3 \&$ isAllowed $=y$

Rizo, H. (1999). Evaluación del docente universitario. Revista Electrónico Interuniversitaria de Formación del Profesorado, 2(1). Recuperado de https://www.aufop.com/aufop/uploaded_files/articulos/1224341864.pdf

Santelices, M., Galleguillos, P., González, J. y Taut, S. (2015). Un estudio sobre la calidad docente en Chile: El rol del contexto en donde enseńa el profesor y 
medidas de valor agregado. Psykhe, 24(1), 1-14. https://doi.org/10.7764/ psykhe.24.1.673

Sistema Nacional de Evaluación, Acreditación y Certificación de la Calidad Educativa - Sineace (2016). Modelo de Acreditación para Programas de Estudios de Educación Superior Universitaria. Recuperado de https://www. sineace.gob.pe/wp-content/uploads/2014/08/Anexo-1-nuevo-modeloprogramas-Resolucion-175.pdf

Strauss, A. y Corbin, J. (2002). Bases de la investigación cualitativa. Técnicas y procedimientos para desarrollas la teoría fundamentada. Medellín, Colombia: Editorial Universidad de Antioquía.

Tumino, M. y Poitevin, E. (2013). Evaluación de la calidad de servicio universitario desde la percepción de estudiantes y docentes: caso de estudio Quality assessment of university service from students and teacher's perceptions: a case study. REICE. Revista Iberoamericana sobre Calidad, 12(2), 63-84. Recuperado de www.rinace.net/reice/

Unesco (1999). Declaración sobrela ciencia y el uso delsabercientifico y programa en pro de la ciencia: marcogeneralde acción. Recuperado de https://unesdoc.unesco. org/ark:/48223/pf0000116994_spa?posInSet=2\&queryId=edc97c2c9b27-44ef-948e-b17b7cc6bf77

Unesco (2009). Conferencia Mundial sobre la Educación Superior - 2009: La nueva dinámica de la educación superior y la investigación para el cambio social y el desarrollo. París, 5-8 de julio de 2009. Recuperado de https://unesdoc. unesco.org/ark:/48223/pf0000183277_spa 